\title{
The Association Between Somatostatin Receptor Ligand and Vitamin B12 Levels in Patients With Acromegaly
}

\author{
Serdar Sahin \\ Gaziosmanpasa Taksim Egitim ve Arastirma Hastanesi \\ Tugce Eskazan \\ Istanbul Universitesi-Cerrahpasa Cerrahpasa Tip Fakultesi \\ Ebru Cicek \\ Istanbul Universitesi-Cerrahpasa Cerrahpasa Tip Fakultesi \\ Yeliz Yagiz Ozoguz \\ Istanbul Universitesi-Cerrahpasa Cerrahpasa Tip Fakultesi \\ Emre Durcan \\ Istanbul Universitesi-Cerrahpasa Cerrahpasa Tip Fakultesi \\ Cem Sulu \\ Istanbul Universitesi-Cerrahpasa Cerrahpasa Tip Fakultesi \\ Hande Mefkure Ozkaya \\ Istanbul Universitesi-Cerrahpasa Cerrahpasa Tip Fakultesi \\ Ali İbrahim Hatemi \\ Istanbul Universitesi-Cerrahpasa Cerrahpasa Tip Fakultesi \\ Pinar Kadioglu ( $\nabla$ kadioglup@yahoo.com ) \\ Istanbul Universitesi-Cerrahpasa Cerrahpasa Tip Fakultesi https://orcid.org/0000-0002-8329-140X
}

\section{Research Article}

Keywords: Acromegaly, octreotide LAR, lanreotide autogel, vitamin B12

Posted Date: August 18th, 2021

DOl: https://doi.org/10.21203/rs.3.rs-806743/v1

License: @ (1) This work is licensed under a Creative Commons Attribution 4.0 International License. Read Full License 


\section{Abstract}

Purpose: Vitamin B12 causes hematologic and neuropsychiatric disorders, so it is important to evaluate it in risky situations. In this study, we aimed to evaluate the association between somatostatin receptor ligands and vitamin B12 levels in patients with acromegaly.

Methods: Patients who were followed up with the diagnosis of acromegaly in the Endocrinology and Metabolism outpatient clinic of Istanbul UniversityCerrahpaşa Medical Faculty were evaluated. Patients were divided into groups according to their somatostatin receptor ligand use status. The groups were evaluated according to their vitamin B12 levels, demographic data, and biochemical parameters.

Results: One hundred fifty-two patients were evaluated. Thirteen patients had vitamin B12 deficiency. The majority (11/13) of patients with vitamin B12 deficiency were patients using somatostatin receptor ligand. In addition, the number of patients with vitamin B12 deficiency who received lanreotide autogel treatment was significantly higher compared with patients who did not use somatostatin receptor ligand $(p=0.011)$. Vitamin B12 levels were higher in patients who received lanreotide autogel treatment than in patients who did not use somatostatin receptor ligand treatment $(p=0.040)$. There was a negative correlation between vitamin B12 levels and lanreotide autogel use time, cumulative lanreotide autogel dose.

Conclusion: It is important to evaluate the level of vitamin B 12 in the follow-up of patients with acromegaly using somatostatin receptor ligand treatment.

\section{Introduction}

Acromegaly is a chronic disease caused by excessive growth hormone (GH) secretion and consequently increased levels of insulin-like growth factor I (IGF-I) [1]. In $95 \%$ of patients, acromegaly is caused by pituitary adenoma, which causes somatotroph cell-derived GH and IGF-1 hypersecretion [2]. Hypersecretion of GH causes high levels of IGF-1, leading to a somatic over-growth, multiple comorbidities, mortality, and physical disfigurement [1]. Morbidity and mortality in patients with acromegaly are mostly due to tumor compression, the elevation of GH/IGF-1, and secondary effects of treatment [3,4]. Mortality is two-fold higher in patients with acromegaly due to the presence of diabetes, hypertension, cardiovascular, cerebrovascular, respiratory and malignancy [5-7].

The primary treatment in patients with acromegaly is surgery, and the transsphenoidal route is generally preferred [1]. Biochemical control rates after surgery range from $32-85 \%$ in patients with acromegaly [8]. Medical treatment is recommended for patients who cannot be controlled after surgery and surgery is not appropriate. Among the medical treatments, somatostatin receptor ligands (SRLs) (octreotide, lanreotide, and pasireotide), cabergoline, pegvisomant, and combination therapies are included [9]. Among the medical treatments, first-generation SRLs [lanreotide autogel and octreotide long-acting release (LAR)] are the first recommended [9]. Common adverse effects of first-generation SRLs include abdominal cramps, flatulence, and diarrhea. In addition, local skin irritation, pain at the injection site, and hair loss are among the rarer adverse effects [1].

Vitamin B12 (Vit B-12) is essential for DNA synthesis and neurologic functions. Active and passive mechanisms play a role in the absorption of Vit B-12. When supraphysiologic amounts of Vit B-12 reach the small intestine, it is directly absorbed passively from the jejunum and ileum. Gastric intrinsic factor (IF) is required for the activation mechanism, and the absorption of the physiologic amount of Vit B-12 in foods occurs in this way [10]. Vit B-12 deficiency can lead to hematologic and neuropsychiatric disorders [10]. One of the rare adverse effects of SRL is Vit B-12 deficiency [11]. Therefore, Vit B-12 deficiency may be important in patients with acromegaly using SRLs. However, there are insufficient data on this subject in the literature. In this study, we aimed to evaluate the association between SRLs and Vit B-12 deficiency in patients with acromegaly.

\section{Materials And Methods}

The medical records of patients with acromegaly followed by the Endocrinology and Metabolism outpatient clinic of Istanbul University-Cerrahpasa, Cerrahpasa Medical Faculty, between 1986 and 2020 were retrospectively reviewed. Patients who were diagnosed as having acromegaly and whose Vit B-12 levels were evaluated were examined. Patients whose Vit B-12 levels were not evaluated; those with a lack of regular follow-ups; patients with a diagnosis of chronic pancreatitis, atrophic gastritis, malabsorption, and inflammatory bowel disease; patients who did not use somatostatin therapy regularly; and those with a history of gastrointestinal surgery were excluded from the study.

All parameters of the patients were analyzed according to the period in which Vit B-12 levels were evaluated. Patients' age, sex, Vit B-12 level, Vit B-12 deficiency status, alcohol consumption and smoking status, proton pump inhibitor (PPI) use, metformin use, use of cabergoline and pegvisomant, total disease duration, GH, IGF-1, IGF-1-ULN values, the biochemical control status, hemoglobin, hematocrit, mean corpuscular volume (MCV), white blood cell (WBC), platelet count, and abdominal imaging were retrospectively analyzed. Octreotide LAR, lanreotide autogel cumulative dose and mean dose every 28 days, and treatment durations were calculated.

Patients with IGF-1 values within the normal range according to age and sex and random GH values less than $1.0 \mathrm{~g} / \mathrm{L}$ were accepted as having controlled disease activity [1]. In discordant cases, patients with no deterioration in clinical and laboratory parameters, and patients with IGF-1 values repeatedly in the normal range in the follow-up period were accepted as having controlled disease activity. GH and IGF-1 were measured in the same laboratory (Supplementary material 1). Vit B-12 levels less than $180 \mathrm{pg} / \mathrm{mL}$ were accepted as deficiency. Vit B-12 was assayed using an electrochemiluminescence immunoassay (ECLIA) method with Roche Cobas e601 systems (Roche, Cobas e 601, Roche Diagnostics GmbH, Mannheim, Germany). GH and IGF-1 were measured in the same laboratory (Supplementary material 1).

The patients were divided into two groups according to the use of SRLs; patients using SRLs and those not using SRLs. The two groups were compared with each other according to age, sex, alcohol, and smoking status, Vit B-12 level, Vit B-12 deficiency, biochemical parameters, biochemical control status, and PPI, metformin, cabergoline, and pegvisomant use. Also, patients were divided into three groups according to the use of SRLs. Group A consisted of patients who do not use SRLs, group B comprised patients using octreotide LAR, and group C constituted patients using lanreotide autogel. These three groups were

Page 2/9 
compared with each other in terms of age, sex, alcohol, and smoking status, Vit-B12 level, Vit B-12 deficiency, biochemical parameters, biochemical control status, and PPI, metformin, cabergoline, and pegvisomant use.

This study was approved by the Local Ethics Committee of Cerrahpasa Faculty of Medicine. The study adhered to the tenets of the Declaration of Helsinki.

\section{Statistical Analysis}

The Statistical Package for the Social Sciences v.22.0 software was used. The distribution of variables was evaluated using the Kolmogorov-Smirnov test. In normally distributed variables, continuous data are given as means \pm standard deviation (SD), in non-normally distributed variables, continuous data are given as median [IQR] and categorical data are given as numbers and percentages. In the comparisons of two groups, the Mann-Whitney $U$ test was performed in the analysis of quantitative, non-normal distribution, and independent data. The independent samples t-test was used in the analysis of quantitative, normal distribution, and independent data. In the comparisons of three groups, for the analysis of quantitative independent data, we used analysis of variance (ANOVA) for data with normal distribution, and in post-hoc analysis, we performed the Bonferroni test. For data with non-normal distribution, we used the Kruskal-Wallis, and Mann-Whitney $U$ test for the analysis of quantitative independent data Bonferroni correction was then performed to account errors due to the multiple comparisons among groups A, B, ad C. The Chi-square test, and, if Chi-square conditions were not be met, Fisher's test was performed in the analysis of qualitative independent data. Statistically significant results were considered as $\mathrm{P}<0.05$. The confidence level was set at $95 \%$.

\section{Results}

In our study, 160 patients with acromegaly were assessed. Three patients were excluded due to atrophic gastritis, one due to terminal ileum resection, and four patients due to irregular SRL treatment use. One hundred fifty-two patients with acromegaly were evaluated, 71 (46\%) of the patients were male and 81 (54\%) were female. All data were evaluated according to the period in which the patients were assessed for Vit B-12. The mean age of the patients was $45.3 \pm 11.8$ years, the mean age at the time of diagnosis was $41.1 \pm 12.0$ years. The mean duration of the disease was 32.5 [range, 16.0-54.7] years. The mean Vit B-12 level was 289.5 [242.0-374.0] pg/mL, 13 (8.5\%) patients had Vit B-12 deficiency. Fifty-nine (38.8\%) patients were not using SRLs, and 93 (61.2\%) patients were using SRLs. Of the patients using SRLs, 59 (38.8\%) were using octreotide long-acting release (LAR) and 34 (22.4\%) were using lanreotide autogel. The cumulative octreotide LAR dose was 990.0 [range 365.0-1990.0] mg, and the mean octreotide LAR dose was 25.0 [range 20.0-30.0] mg/28 days. The cumulative lanreotide autogel dose was 2400.0 [range 1064.9-3757.2] mg, and the mean lanreotide autogel dose was 101.5 [range $90.0-114.0$ ] mg/28 days. The median [IQR] hemoglobin, hematocrit, MCV, WBC, platelet count values were 13.0 [range, 12.0-14.0] g/dL, 39.0\% [range, 36.1-41.0], 85.0 [range, 82.0-87.2] $\mathrm{fl}, 6800$ [range, 5800-8300] mcL, and 249,000 [range, 200,000-292,000] mcL, respectively. The alcohol consumption and smoking data of 111 patients were obtained. Seven (6\%) patients consumed alcohol, and 36 (32\%) patients smoked. One (7\%) of 13 patients with Vit B-12 deficiency smoked, while none of them consumed alcohol.

Anemia was present in $53(34.9 \%)$ patients, and of these, 15 (28.3\%) were microcytic, 38 (71.7\%) were normocytic; there was no macrocytic anemia. Eight (61.5\%) of 13 patients with Vit B-12 deficiency had anemia. Four (50\%) of these patients had microcytic anemia and four (50\%) had normocytic anemia. None of the patients had macrocytic anemia. Thirty-three (21.7\%) patients were using cabergoline, three (2.0\%) were using pegvisomant, 11 (7.2\%) were using PPIs, and $27(17.8 \%)$ patients were using metformin. One (7\%) of 13 patients with Vit B-12 deficiency were using PPIs, while none of them were using metformin. Abdominal imaging was present in $102(67.1 \%)$ patients. Of these patients, 97 (63.8\%) had abdominal ultrasonography, three (2\%) had abdominal magnetic resonance imaging (MRI), two (1.3\%) had abdominal computed tomography (CT) imaging. None of these patients had any findings of pancreatitis. Fifty-three patients had upper gastrointestinal system endoscopy. None of these patients had any findings of atrophic gastritis. Of the 13 patients with Vit B-12 deficiency, seven (53\%) had abdominal imaging, and none had signs of pancreatitis. Upper gastrointestinal endoscopy and endoscopic biopsy were performed in all patients with Vit B-12 deficiency. Only one patient had antrum biopsy, while the others had both antrum and corpus biopsy. Atrophic gastritis was not detected in any of the patients. General features of patients with Vit B12 deficiency are presented in Table 1. 
Table 1

The general characteristics of patients with vitamin B12 deficiency

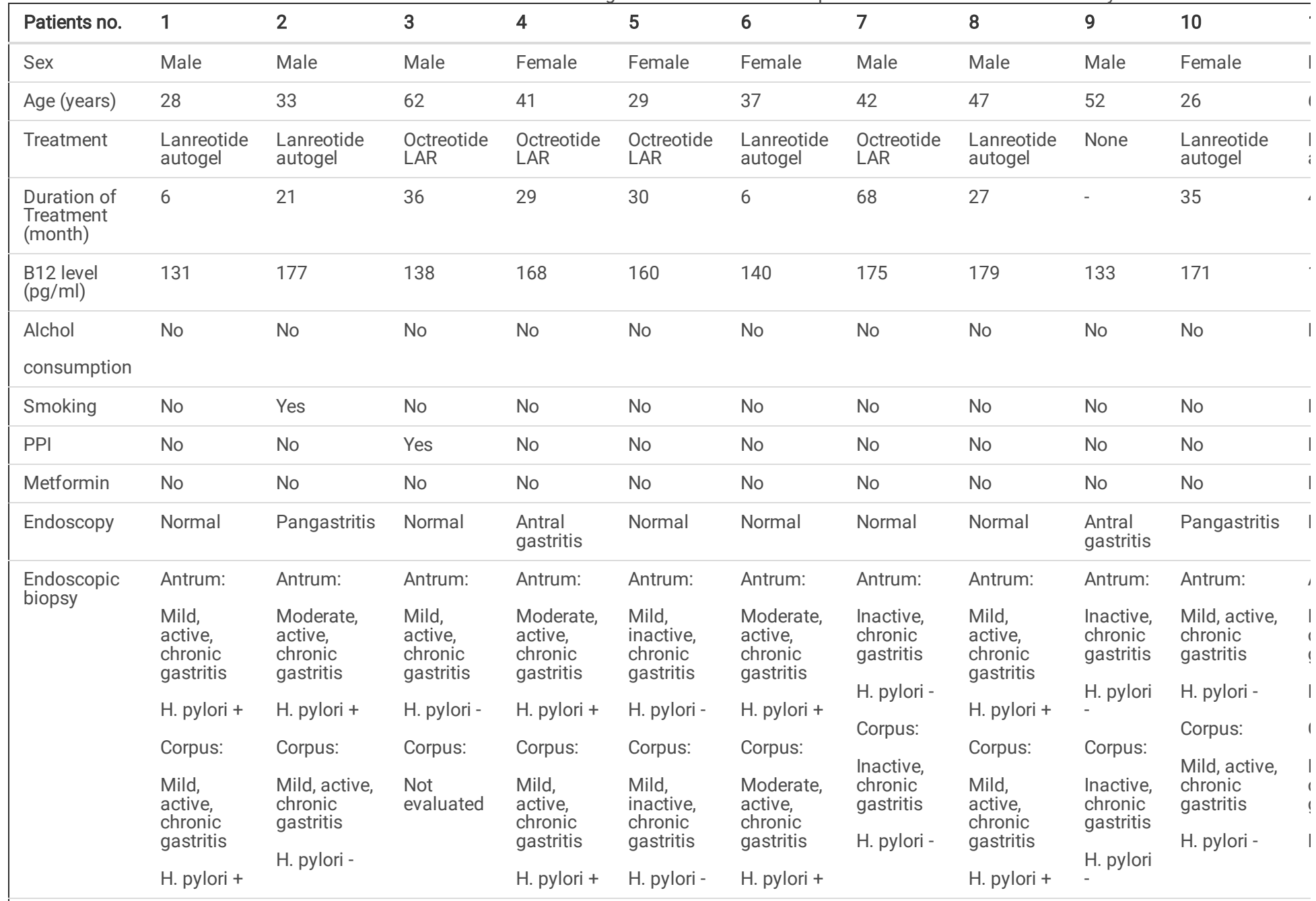

PPI: Proton pump inhibitors

The comparison of SRL treatment $(+)$ vs. SRL treatment $(-)$

No significant difference was found among the groups in terms of sex and age ( $p=0.631$ and $p=0.666$, respectively). There was no significant difference between the groups in terms of alcohol consumption and smoking ( $p=0.701$ and $p=0.911$, respectively). Vit B-12 deficiency was present in 11 ( $11.8 \%$ ) patients in the SRL treatment + group and $2(3.4 \%)$ patients in the SRL treatment - group, which was not statistically significantly different $(p=0.070)$. In addition, the median Vit B-12 level was 288.0 [range, 223.0-373.0] pg/mL in the SRL treatment (+) group and 297.0 [range, 246.0-375.0] pg/mL in the SRL treatment (-) group; the difference was not statistically significant $(p=0.268)$. Hemoglobin and hematocrit values were significantly lower in the SRL treatment $(+)$ groups $(p=0.019$ and $p=0.014$, respectively). However, no significant difference was found in terms of the presence of anemia $(p=0.090)$. There was no statistical significance between the groups in terms of MCV levels $(p=0.539)$. In terms of disease duration, the median value was 40.0 [range, $23.5-70.0$ ] months in the SRL treatment (+) group and 23.0 [range, 12.0-37.0] months in the SRL treatment (-) group, which was statistically significantly different ( $p<$ 0.001). There was no difference between the groups in terms of GH, IGF-1, IGF-1-ULN, and disease control $(p=0.506, p=0.852, p=0.986$, and $p=0.576$, respectively). No significance was found between the groups in terms of the use of PPIs, metformin, and pegvisomant $(p=0.204, p=0.280$, and $p=0.283$, respectively), whereas the use of cabergoline was significantly higher in the group using SRLs $(p<0.001)$. The comparison of groups are presented in Table 2 . 
Table 2

The comparison of SRL treatment + group and SRL treatment - group

\begin{tabular}{|llll|}
\hline \multicolumn{4}{|c}{ The comparison of SRL treatment + group and SRL treatment - group } \\
\hline & SRL treatment - & SRL treatment + & p \\
\hline Gender F (\%) / M (\%) & $30(50.8 \%) / 29(49.2 \%)$ & $51(54.8 \%) / 42(45.2 \%)$ & 0.631 \\
\hline Age means \pm SD (year) & $45.8 \pm 12.1$ & $44.9 \pm 11.7$ & 0.666 \\
\hline Alcohol use (n (\%)) & $2(4.5 \%)$ & $5(7.5 \%)$ & 0.701 \\
\hline Smoking (n (\%)) & $14(31.8 \%)$ & $22(32.8 \%)$ & 0.911 \\
\hline Vitamin B12 level median [IQR] (pg/ml) & $297.0[246.0-375.0]$ & $288.0[223.0-373.0]$ & 0.268 \\
\hline Vitamin B12 deficiency (n (\%)) & $2(3.4 \%)$ & $11(11.8 \%)$ & 0.070 \\
\hline Hemoglobine median [IQR] (g/dl) & $13.5[12.6-14.4]$ & $12.9[12.0-13.8]$ & 0.016 \\
\hline Hematocrite median [IQR] (\%) & $39.8[37.2-42.9]$ & $38.6[35.5-40.9]$ & 0.011 \\
\hline MCV median [IQR] (fl) & $85.0[82.0-88.0]$ & $84.1[82.0-87.0]$ & 0.539 \\
\hline PPI use (n (\%)) & $2(3.4 \%)$ & $9(9.4 \%)$ & 0.204 \\
\hline Metformin use (n (\%)) & $8(13.6 \%)$ & $19(20.4 \%)$ & 0.280 \\
\hline F: Female, M: Male, SRL: Somatostatin receptor ligand, MCV: Mean corpuscular volume & \\
\hline PPI: Proton pump inhibitor & & & \\
\hline
\end{tabular}

In the correlation analysis, a negative correlation was found between Vit B-12 levels and the duration of SRL use $(p=0.030 r=-0.232)$. However, no correlation was found between Vit B-12 levels and age and total disease duration.

\section{The comparison of groups A (SRL treatment -), B (Octreotide LAR +), and C (Lanreotide autogel +)}

There was no statistically significant difference between the groups in terms of sex and age $(p=0.464$ and $p=0.162$, respectively). The disease duration was found to be significantly lower in group A (SRL treatment -$)(p<0.05)$. There was no significant difference between the groups in terms of alcohol consumption and smoking ( $p=0.533$ and $p=0.178$, respectively). In terms of Vit B-12 deficiency, the number of patients with Vit B-12 deficiency was found to be significantly higher in group $C$ (lanreotide autogel + ) than in group A (SRL treatment -$)(p=0.011)$. Although there was no statistically significant difference, the number of patients with Vit B-12 deficiency was higher in group C (lanreotide autogel + ) than in group B (octreotide LAR +$)(p=0.091)$. When analyzed in terms of Vit B-12 levels between the groups, Vit B-12 levels were found to be significantly lower in patients using lanreotide autogel (group C) than in patients not using SRL treatment (group A) ( $p=0.040)$. There was no significant difference between the groups in terms of anemia and MCV. Although there was a significant difference in terms of hemoglobin and hematocrit ( $p=0.050$ and $p=0.039$, respectively), the statistical significance disappeared in pairwise comparisons. There was no significant difference between the groups in terms of pegvisomant, $P P I$, and metformin use $(p=0.442, p=0.221$ and $p=0.141$, respectively). However, the use of cabergoline was found to be significantly lower in group B than in the other groups ( $<<0.001$ and $p<0.001$, respectively). There was no significant difference between the groups in terms of GH, IGF-1, IGF-1-ULN, and disease control $(p=0.622, p=0.752, p=0.785$, and $p=0.706$ respectively). The comparison of groups A-B-C are presented in Table 3. 
Table 3

The comparison of Groups A-B-C

\begin{tabular}{|c|c|c|c|c|}
\hline & Group A & Group B & Group C & $\mathrm{p}$ \\
\hline Gender F (\%) / M (\%) & $30(50.8) / 29(49.2)$ & $35(59.3) / 24(40.7)$ & $16(47.1) / 18(52.9)$ & 0.464 \\
\hline Age means $\pm S D$ (year) & $45.8 \pm 12.1$ & $46.0 \pm 10.4$ & $41.9 \pm 13.3$ & 0.162 \\
\hline Alcohol use (n (\%)) & $2(4.5)$ & $2(4.9)$ & $3(11.5)$ & 0.533 \\
\hline Smoking (n (\%)) & $14(31.8)$ & $10(24.4)$ & $12(46.2)$ & 0.178 \\
\hline The disease duration (month) & $23.0[12.0-37.0]$ & $42.0[24.0-115.0]^{\mathrm{a}}$ & $37.0[17.0-47.7]$ & $<0.001$ \\
\hline The duration of SRL use (month) & - & $31.0[15.5-75.0]$ & $23.5[11.0-36.5]$ & 0.047 \\
\hline B12 level median [IQR] (pg/ml) & $297.0[246.0-375.0]^{\mathrm{b}}$ & $290.0[228.0-375.0]$ & $268.0[194.0-329.0]$ & 0.105 \\
\hline B12 deficiency (n (\%)) & $2(3.4)$ & $4(6.8)$ & $7(20.6)^{c}$ & 0.014 \\
\hline Hemoglobine median [IQR] $(\mathrm{g} / \mathrm{dl})^{\mathrm{d}}$ & $13.5[12.6-14.4]$ & $12.9[11.9-13.7]$ & $13.0[11.9-14.0]$ & 0.050 \\
\hline Hematocrite median [IQR] (\%) & $39.8[37.2-42.9]$ & $38.6[35.4-40.2]$ & $38.2[35.8-41.0]$ & 0.039 \\
\hline MCV median [IQR] (fl) & $85.0[82.0-88.0]$ & $84.6[82.0-87.1]$ & $84.0[82.1-87.0]$ & 0.772 \\
\hline PPI use (n (\%)) & $2(3.4)$ & $7(11.9)$ & $2(5.9)$ & 0.221 \\
\hline Metformin use (n (\%)) & $8(13.6)$ & $15(25.4)$ & $4(11.8)$ & 0.141 \\
\hline \multicolumn{5}{|c|}{ F: Female, M: Male, SRL: Somatostatin receptor ligand, MCV: Mean corpuscular volume, PPI: Proton pump inhibitor } \\
\hline \multicolumn{5}{|c|}{$\begin{array}{l}{ }^{b} \text { There was no statistically significant difference among groups, but a significant difference was found between Group A and Group C in pairwise } \\
\text { comparisons }(p=0.040)\end{array}$} \\
\hline \multicolumn{5}{|l|}{${ }^{\circ}$ Group C vs. Group A $(p=0.011)$} \\
\hline
\end{tabular}

In the correlation analysis, a negative correlation was found between Vit B-12 level and cumulative lanreotide autogel dose ( $p=0.048, r=-0.342)$, the duration of lanreotide autogel use $(p=0.035, r=-0.363)$ in group $C$ (lanreotide autogel). However, no correlation was found between Vit B-12 levels and age, total disease duration, and dose (mg/28 days). In group B, there was no significant correlation between Vit B-12 levels and these parameters (Table 4). 
Table 4

The correlation analyses in the Group B and Group C

\begin{tabular}{|c|c|}
\hline Group B (Octreotide LAR +) & B12 level \\
\hline \multirow[t]{2}{*}{ The mean Octreotide LAR dose (mg/28 days) } & $p=0.934$ \\
\hline & $r=-0.011$ \\
\hline \multirow[t]{2}{*}{ The cumulative Octreotide LAR dose (mg) } & $p=0.140$ \\
\hline & $r=-0.205$ \\
\hline \multirow[t]{2}{*}{ The duration of Octreotide LAR use } & $p=0.106$ \\
\hline & $r=-0.224$ \\
\hline \multirow[t]{2}{*}{ Age } & $p=0.460$ \\
\hline & $r=0.098$ \\
\hline \multirow[t]{2}{*}{ The total disease duration } & $p=0.229$ \\
\hline & $r=-0.159$ \\
\hline \multicolumn{2}{|l|}{ Group C (Lanreotide autogel +) } \\
\hline \multirow[t]{2}{*}{ The mean Lanreotide autogel dose (mg/28 days) } & $p=0.880$ \\
\hline & $r=0.027$ \\
\hline \multirow[t]{2}{*}{ The cumulative Lanreotide autogel dose (mg) } & $p=0.048$ \\
\hline & $r=-0.342$ \\
\hline \multirow[t]{2}{*}{ The duration of Lanreotide autogel use } & $p=0.035$ \\
\hline & $r=-0.363$ \\
\hline \multirow[t]{2}{*}{ Age } & $p=0.804$ \\
\hline & $r=0.044$ \\
\hline \multirow[t]{2}{*}{ The total disease duration } & $p=0.028$ \\
\hline & $r=-0.376$ \\
\hline
\end{tabular}

\section{Discussion}

The majority of patients with Vit B-12 deficiency were in the SRL (+) treatment group. The number of patients with Vit B-12 deficiency was found to be significantly higher in the group that received lanreotide autogel treatment (group C) compared with the patients who did not receive SRL treatment (group A). In addition, Vit B-12 levels were found to be significantly lower in patients who received lanreotide autogel treatment compared with patients who did not receive SRL treatment. Considering the factors affecting Vit B-12 deficiency, a negative correlation was found between the duration of lanreotide autogel treatment, cumulative lanreotide autogel dose and Vit B-12 levels. There was no difference between the groups in terms of PPI, metformin, alcohol consumption, and smoking. Hemoglobin and hematocrit levels were found to be significantly lower in patients treated with SRLs.

Although there are data that somatostatin analogs cause Vit B-12 deficiency, there is not robust studies in the literature on this issue. In an early study conducted by Plockinger et al., 10 patients with acromegaly treated with subcutaneous octreotide were prospectively evaluated. In patients with normal Vit B12 levels at the initiation of octreotide treatment, Vit B-12 levels decreased over time. When the treatment period was prolonged, Vit B-12 levels fell below the normal limits in four patients [12]. In our study, although the level of Vit B-12 was not statistically significant, it was found to be lower in the group with SRL treatment. In addition, the number of patients with deficient Vit B-12 levels in patients who received lanreotide autogel treatment was found to be significantly higher than in those who did not receive SRL treatment. In addition, Vit B-12 levels were lower in patients who received lanreotide autogel treatment compared with patients who did not receive SRL treatment. However, this difference was not detected in the group receiving octreotide LAR treatment. Interestingly, although the significance was found in the group receiving lanreotide autogel treatment, the duration of octreotide LAR treatment was significantly longer than lanreotide autogel treatment.

The mechanism by which somatostatin analogues cause Vit B-12 deficiency is also unclear because there has not been much research on this issue. There are two opinions on this subject. First, somatostatin analogs can directly inhibit the release of IF, leading to Vit B-12 deficiency [12]. The other view is that somatostatin analogs may cause Vit B-12 deficiency by preventing acid secretion from gastrin-secreting cells, creating hypochlorhydria [12, 13]. We cannot comment on this subject because our study is retrospective, but this issue needs large, and prospective studies.

Vit B-12 deficiency causes megaloblastic anemia by disrupting DNA synthesis. In addition to anemia, it can cause a decrease in all blood cells (pancytopenia) [14]. In our study, none of the patients with Vit B-12 deficiency had macrocytic anemia or pancytopenia. There was no difference between the groups in terms of MCV. There is not much information on this subject in the literature. In the study of Plockinger et al., although Vit B-12 deficiency was observed in patients receiving octreotide treatment, no hematologic change was detected in any patients [12]. 
There are several limitations to our study. The IF levels of the patients could not be evaluated because of our study's retrospective design, and pre-SRL treatment Vit B-12 levels were unknown. The course of Vit B-12 could not be evaluated in patients receiving SRL treatment. In addition, neurologic examinations of patients with Vit B-12 deficiency could not be performed.

In conclusion, Vit B-12 levels have an important place in patient follow-up due to their hematologic and neurologic consequences. Evaluation and determination of Vit B-12 levels in the follow-up of patients with acromegaly receiving SRL treatment allow us to perform replacement at the appropriate time. Before pancytopenia and neurological deficit occur, initiation of Vit B-12 replacement can provide positive results in terms of time and economic burden for the patient and healthcare providers.

\section{Declarations}

\section{Funding:}

This research did not receive any specific grant from any funding agency in the public, commercial or not-for-profit sector.

\section{Compliance with ethical standards}

\section{Conflict of interest:}

The authors declare that they have no conflict of interest.

\section{Ethical approval:}

This study was approved by the Local Ethics Committee of Istanbul University-Cerrahpasa, Cerrahpasa Medical Faculty. The study adhered to the tenets of Helsinki.

\section{Research involving human and animal participants:}

This article does not contain any studies with animals performed by any of the authors.

\section{References}

1. L. Katznelson, E. R. Laws, Jr, S. Melmed, M. E. Molitch, M.H. Murad, A. Utz, J. A. H. Wass. Acromegaly: An endocrine society clinical practice guideline. Journal of Clinical Endocrinology and Metabolism. 99 (11):3933-3951 (2014). https://doi.org/10.1210/jc.2014-2700

2. S. Melmed. Acromegaly pathogenesis and treatment. Journal of Clinical Investigation. 119 (11):3189-3202 (2009). https://doi.org/10.1172/JCl39375

3. A. Colao, D. Ferone, P. Marzullo. Systemic Complications of Acromegaly: Epidemiology, Pathogenesis, and Management. Endocrine Reviews. 25 (1):10252 (2004). https://doi.org/ 10.1210/er.2002-0022

4. S. Melmed, F. F. Casanueva, A. Klibanski, M. D. Bronstein, P. Chanson, S. W. Lamberts, C. J. Strasburger, J. A. H. Wass, A. Giustina. A consensus on the diagnosis and treatment of acromegaly complications. Pituitary. 16 (3):294-302 (2013). https://doi.org/10.1007/s11102-012-0420-x

5. I. M. Holdaway, M.J. Bolland, G.D. Gamble. A meta-analysis of the effect of lowering serum levels of GH and IGF-I on mortality in acromegaly. European Journal of Endocrinology. 159 (2):89-95 (2008). https://doi.org/10.1530/EJE-08-0267

6. O.M. Dekkers, N.R. Biermasz, A.M. Pereira, J.A. Romijn, J.P. Vandenbroucke. Mortality in acromegaly: A metaanalysis. Journal of Clinical Endocrinology and Metabolism. 93 (1):61-7 (2008). https://doi.org/10.1210/jc.2007-1191

7. M. E. Sughrue, E. F. Chang, R. A. Gabriel, M. K. Aghi, L. S. Blevins. Excess mortality for patients with residual disease following resection of pituitary adenomas. Pituitary. 14 (3):276-283 (2011). https://doi.org/10.1007/s11102-011-0308-1

8. N. Agrawal, A.G. loachimescu. Prognostic factors of biochemical remission after transsphenoidal surgery for acromegaly: a structured review. Pituitary. 23 (5):582-594 (2020). https://doi.org/10.1007/s11102-020-01063-x.

9. S. Melmed, M. D. Bronstein, P. Chanson, A. Klibanski, F. F. Casanueva, J. A. H. Wass, C. J. Strasburger, A. Luger, D.R. Clemmons, A. Giustina. A Consensus Statement on acromegaly therapeutic outcomes. Nature Reviews Endocrinology. 14 (9):552-561 (2018). https://doi.org/10.1038/s41574-018-0058-5

10. R.C. Oh, D.L. Brown. Vitamin B12 deficiency. American Family Physician 1;67(5):979-86 (2003)

11. S.M. Consortium Use of Somatostatin Analogues in Adult Patients with Acromegaly Shared Care Guidelines. 1-16 (2011)

12. U. PLÖCKINGER, D. DIENEMANN, H.J. QUABBE. Gastrointestinal Side-Effects of Octreotide during Long Term Treatment of Acromegaly. The Journal of Clinical Endocrinology \& Metabolism. 71 (6):1658-1662 (1990). https://doi.org/10.1210/jcem-71-6-1658

13. G.J. Krejs. Physiological role of somatostatin in the digestive tract: Gastric acid secretion, intestinal absorption, and motility. Scandinavian Journal of Gastroenterology 119:47-53 (1986). https://doi.org/10.3109/00365528609087431

14. R. Green, L.H. Allen, A.L. Bjørke-Monsen, A. Brito, J.L. Guéant, J.W. Miller, A.M. Molloy, E. Nexo, S. Stabler, B.H. Toh, P. M. Ueland, C. Yajnik. Vitamin B12 deficiency. Nature Reviews Disease Primers. 29;3:17040 (2017). https://doi.org/10.1038/nrdp.2017.40

\section{Supplementary Files}

This is a list of supplementary files associated with this preprint. Click to download. 
- Supplementarymaterial.docx

Page 9/9 